\title{
Gemeinsinn und Solidarität: Motivation und Wirkung
}

\section{von Freiwilligenarbeit}

Zusammenfassung: Freiwilligenarbeit ist eine zentrale Säule der Bürgergesellschaft und fördert wechselseitige Teilhabe. In diesem Text wird ein Überblick über die psychologische Motivforschung zur Freiwilligenarbeit gegeben. Es werden konkrete Empfehlungen abgeleitet, wie sich entsprechende Commitments und Verhaltensweisen fördern lassen.

Schlüsselwörter: Freiwilligenarbeit, bürgerschaftliche Engagements, Solidarität, Verantwortung, Gerechtigkeit

\section{Public Spirit and Solidarity: Motivation and Impact of Volunteering}

Abstract: Volunteering is a key pillar of our civil society and promotes mutual social inclusion. This text gives an overview on psychological motive research on volunteering. Specific recommendations are derived, how relevant commitments and decisions can be promoted.

Keywords: Voluntary work, civic engagement, solidarity, responsibility, justice

\section{$1 \quad$ Einstieg}

Unsere Zivil- bzw. Bürgergesellschaft ist auf freiwillige Engagements ihrer Bürgerinnen und Bürger angewiesen. Sie ermöglichen gesellschaftliche Partizipation, fördern gesellschaftliche Integration und bedeuten zugleich hohe ökonomische Vorteile für die Gesellschaft. Letzteren können durchaus auch Nachteile gegenüberstehen, wie die Gefahr der Instrumentalisierung der freiwilligen Engagements, die Entlastung aus sozialstaatlicher Verantwortung und Fürsorge ${ }^{1}$ sowie einer Veränderung der Motivlagen. ${ }^{2}$ Zweifelsfrei führen sie jedoch zu wechselseitiger Teilhabe und sind eine große Säule der Bürgergesellschaft. Dies zeigt sich in ganz besonderer Weise in Zeiten von Krisen, wie der Flüchtlingsbewegung oder zuletzt in der Corona-Krise: In kürzester Zeit entstand in den ersten Wochen nach Mitte März 2020 in Deutschland eine Welle der Solidarität, für die sich in der gesamten Breite gesellschaftlichen Lebens vielfältige Beispiele finden, von der Nachbarschaftshilfe über

\footnotetext{
${ }^{1}$ Röbke (2012).

2 Wehner und Güntert (2015).
} 
Preprints 2021 der Zeitschrift BIBLIOTHEK - Forschung und Praxis, BFP.2020.0103 Kals, Strubel und Güntert

Dies ist ein Preprint $(\boldsymbol{c c})$ EY-NG-ND. Die endgültige Publikationsfassung erscheint beim Verlag De Gruyter unter https://www.degruyter.com/view/j/bfup

finanzielle Solidaritätsprojekte bis hin zu digitalen Hilfsangeboten im Bildungsbereich, etwa, um das Home-Schooling zu unterstützen.

All diese Engagements sind Ausdruck von Solidarität und damit der Bereitschaft, einen Beitrag für die Gemeinschaft zu leisten - und zwar unabhängig von etwaigen Eigeninteressen und eigenem Nutzen. Sie werden in ihrer Ausprägung aber auch in ihrer gesellschaftlichen Wertschätzung und Sichtbarkeit durch die aktuellen Umstände in starkem Maße gefördert. Auch aufgrund grundlegender gesellschaftlicher Veränderungsprozesse, wie demographischem Wandel oder Digitalisierung, gewinnen sie stetig an Bedeutung. So bestätigen die repräsentativen, regelmäßig durchgeführten Freiwilligensurveys, dass der Anteil der Engagierten in der allgemeinen Bevölkerung insgesamt hoch ist und stetig zunimmt. Aktuell liegt er bei 43,6 Prozent. $^{3}$ Die Engagements werden in vielfältigen Bereichen erbracht, sie sind jedoch über die verschiedenen Bevölkerungsgruppen ungleich verteilt und hängen u.a. von Alter und Bildungsstand ab. Zudem zeigt sich, dass viele Menschen, die bislang nicht engagiert sind, grundsätzlich bereit wären, sich ebenfalls zu engagieren. Somit liegt hier noch viel Potential brach, um mehr Menschen für ein solches Engagement zu gewinnen.

Um dieses Potential zu nutzen und effiziente Strategien zu entwickeln, vorhandene Bereitschaften in tatsächliche Engagements umzusetzen und kurzfristige Engagements zu stabilisieren, ist psychologisches Wissen über die Motivstrukturen aber auch über Hürden der Engagements notwendig: Wie kommen solidarische und der Gemeinschaft zugutekommende Verhaltensweisen und Engagements des Einzelnen zustande? Wodurch werden sie gefördert, wodurch gehemmt? Welche Ableitungen lassen sich aus diesem Wissen treffen, um freiwillige Engagement zu fördern? Diese Fragestellungen gehen weit über repräsentative Befragungsergebnisse hinaus und stehen im Fokus des vorliegenden Artikels. Dazu sei zunächst die Vielfalt freiwilliger Engagements und ihrer Bezeichnungen betrachtet.

\section{Die Vielfalt freiwilliger Engagements und ihrer Bezeichnungen}

Es gibt innerhalb der Angewandten Sozialpsychologie eine lange Forschungstradition zu altruistischem Handeln. Dabei wurde in unterschiedlichsten Handlungsfeldern nachgewiesen, dass Motive der Verantwortung und Gerechtigkeit unabhängig von und über Eigeninteresse hinaus von Bedeutung sind. ${ }^{4}$ Ein besonders starker Forschungsstrang hat sich zu spontanen Engagements und zum Hilfehandeln herausgebildet. ${ }^{5}$ Hieraus entstand das Forschungsfeld zur Freiwilligenarbeit

\footnotetext{
${ }^{3}$ Simonson et al. (2016).

${ }^{4}$ Gollwitzer et al. (2013).

${ }^{5}$ Dovidio et al. (2006).
} 
Preprints 2021 der Zeitschrift BIBLIOTHEK - Forschung und Praxis, BFP.2020.0103 Kals, Strubel und Güntert

Dies ist ein Preprint $(\boldsymbol{c c})$ EY-NG-ND. Die endgültige Publikationsfassung erscheint beim Verlag De Gruyter unter https://www.degruyter.com/view/j/bfup

(„,volunteering“) bzw. zum bürgerschaftlichen Engagement, das im Zentrum des vorliegenden Artikels steht. Dabei geht es um soziale Engagements im Sinne mittel- und langfristiger Einlassungen auf verschiedene Zielfelder, wie caritative Hilfe, Umwelt- und Gesundheitsschutz, Bildung, Kultur und Sport, Rechtspflege oder Politik. Dabei zeigt sich ein Strukturwandel, bei dem klassische Formate, wie das Ehrenamt, zunehmend durch neue Formen bürgerschaftlichen Engagements ersetzt werden. ${ }^{6}$

Für dieses Engagement finden sich in der Literatur verschiedene Begriffsvarianten, wie Freiwilligenarbeit, frei-gemeinnützige Tätigkeit, Ehrenamt oder auch Volunteering. Gemeinsam sind diesen Begrifflichkeiten folgende definitorische Merkmale: ${ }^{7}$

- „Die angesprochene Tätigkeit ist freiwillig und autonom, selbst oder institutionell organisiert, aber nicht privat.

- Sie ist öffentlich sichtbar, wirksam und nachhaltig, steht aber nicht unter staatlicher Regie und Kontrolle.

- Sie dient (auch) der Wertschöpfung, folgt aber nicht nur einer klassischen ökonomischen Logik, sondern kennzeichnet den Übergang zur Sorgeökonomie.

- Sie ist unbezahlt, lässt sich aber monetarisieren und wird oftmals aufwandsentschädigt oder zunehmend auch geringfügig bezahlt."

Entsprechend dieser Merkmale sind bürgerschaftliche Engagements als eigenständige

Handlungskategorie zu verstehen, die jedoch Bezüge zu prosozialem, verantwortlichem und Hilfehandeln haben. ${ }^{5}$

\section{Die Überwindung der Dichotomie „Eigennutz“ versus „Altruismus“}

In vielen sozialpsychologischen Texten, vor allem in der mittlerweile als klassisch zu bezeichnenden Forschung zu altruistischem Handeln bzw. zu Hilfehandeln, findet sich eine Dichotomisierung von „Altruismus“ versus „Egoismus“. Sie wurde auch in der frühen sozialpsychologischen Freiwilligenforschung aufgegriffen. ${ }^{8}$ Die Ausgangsfrage ist dabei, warum Menschen altruistisch handeln und z. B. in Notsituationen eingreifen, um anderen zu helfen und dabei z. B. eigene Risiken, Gefahren und Belastungen in Kauf nehmen. Ein solches Verhalten wird als „altruistisch“ bezeichnet und steht dem Menschenbild des „homo oeconomicus", der ausschließlich aufgrund eigennütziger Motive handelt, kontrastierend entgegen. Damit geht es um die grundsätzliche Frage: Wie zweckrational bzw. wie „eigennützig“ ist der Mensch in seinem Handeln? Das Modell vom Homo oeconomicus als "rationaler Egoist, der in sozialen Interaktionen seinen subjektiven Nutzen

\footnotetext{
${ }^{6}$ Simonson et al. (2016).

${ }^{7}$ Wehner et al. (2015).

${ }^{8}$ Vgl. Wehner und Güntert (2015).
} 
Preprints 2021 der Zeitschrift BIBLIOTHEK - Forschung und Praxis, BFP.2020.0103 Kals, Strubel und Güntert

Dies ist ein Preprint $(\boldsymbol{c c})$ EY-NG-ND. Die endgültige Publikationsfassung erscheint beim Verlag De Gruyter unter https://www.degruyter.com/view/j/bfup

maximieren möchte und sich dabei zweckrational verhält" ${ }^{9}$ ist dabei die Grundannahme der klassischen ökonomischen Theorie.

In den letzten Jahrzehnten fand jedoch eine sehr breite und kontroverse Diskussion über diese Grundannahme von Eigennutz als dominantes oder gar einziges Motiv menschlichen Handelns statt, denn sie hat theoretische, empirische und gesellschaftspolitische Restriktionen: ${ }^{10}$ In theoretischer Hinsicht widerspricht es wissenschaftlicher Vorgehensweise, die Existenz von nur einem Motiv zu postulieren. Dies ist wissenschaftlich unproduktiv und führt letztlich zu Zirkelschlüsseln, da die Beweggründe jeglichen menschlichen Handelns auf Eigennutz zurückgeführt werden. Als Hilfskonstruktion zur Erklärung offenkundig altruistischen Handelns wurde dabei auch von „verdecktem Eigennutz“ gesprochen, wie die Steigerung des eigenen Selbstwerts, der sich dann hinter solchem Handeln verbergen kann. Empirisch getestet wurden diese Annahmen jedoch selten; meist wurden sie aufgrund der Befundlage post hoc formuliert. Studien, in denen eine Testung von verdecktem Eigennutz stattfand, zeigen hingegen, dass auch dieser nicht relevant ist, so beispielsweise in der Motivforschung zu ökologischen Engagements. ${ }^{11}$ Auch, wenn man das Beispiel der breiten und vielfältigen bürgerschaftlichen Engagements im Kontext der Corona-Krise heranzieht, bedarf es großer Kreativität, um die vielen Engagements jeweils ausschließlich oder primär als eigennützig motiviert zu interpretieren: Warum sollte unter dieser Annahme beispielsweise der junge Student über Wochen hinweg die Einkäufe für die betagte und nicht betuchte Nachbarin übernehmen? Wir können den Fall sogar so konstruieren, dass gar keine direkte Kommunikation, kein direkter Dank und kein Erleben von Freude im direkten sozialen Kontakt möglich ist. Alternative Handlungsmotive für das studentische Engagement in Form sozialer Verantwortung sind hier wahrscheinlicher und stehen in Einklang mit zahlreichen Befunden zu den Motiven, die im Bereich caritativen Engagements bestätigt worden sind. ${ }^{12}$

Schließlich ist es auch gesellschaftspolitisch äußerst problematisch, wenn die Norm etabliert wird, dass Menschen in Entscheidungssituationen jene Option wählen, die ihren eigenen Nutzen maximieren. Durch die Erwartung, dass es angemessen wäre, eigennützig zu handeln wird das Handeln anderer Menschen häufiger als eigennützig motiviert wahrgenommen und eigenes eigennütziges Verhalten gefördert. Eigennütziges Handeln wird dadurch immer stärker gesellschaftsfähig und sozial akzeptiert. Eine Welle der Solidarität und des gemeinschaftlichen

\footnotetext{
${ }^{9}$ Miller (1994) 6.

${ }^{10}$ Kals (1999).

${ }^{11}$ Kals et al. (2001).

${ }^{12}$ Strubel und Kals (2016).
} 
Preprints 2021 der Zeitschrift BIBLIOTHEK - Forschung und Praxis, BFP.2020.0103 Kals, Strubel und Güntert

Dies ist ein Preprint $(\boldsymbol{c c})$ EY-NG-ND. Die endgültige Publikationsfassung erscheint beim Verlag De Gruyter unter https://www.degruyter.com/view/j/bfup

Sorgens und Handelns, wie es zumindest zu Beginn der Corona-Krise zu beobachten gewesen war, wird dadurch erschwert.

Aus theoretischer, empirischer und gesellschaftspolitischer Sicht macht es daher Sinn, von einem Motivpluralismus auszugehen, der menschlichem Handeln zugrunde liegt. Die Frage, welche Motive dabei bedeutsam sind, ist nicht auf der Ebene eines theoretischen Diskurses, sondern durch empirische Daten zu beantworten. Dazu sind verschiedene Motive theoretisch abzuleiten, a priori zu formulieren und anhand entsprechender methodischer Standards zu testen. Das nachfolgende Modell bietet hierzu den geeigneten theoretischen Rahmen.

\section{Multifaktorielles Modell zur Erklärung bürgerschaftlicher Engagements}

Im Zentrum des Modells stehen bürgerschaftliche Engagementbereitschaften und tatsächliche Engagements als zu erklärende Kriterien. Das Modell bildet eine Zusammenschau empirischer Befunde ab, die u.a. auf vielfältigen Kooperationen mit den Kollegen Theo Wehner und Stefan Güntert aus der Zürcher Arbeitsgruppe beruhen (vgl. Abb. 1). Die bürgerschaftlichen Engagements können in unterschiedlichen Handlungsfeldern erbracht werden; die Bereitschaften zu diesen Engagements beziehen sich nicht auf einzelne Handlungen, sondern sind als grundsätzliche Einlassungen auf die jeweiligen Zielfelder des Engagements gefasst.

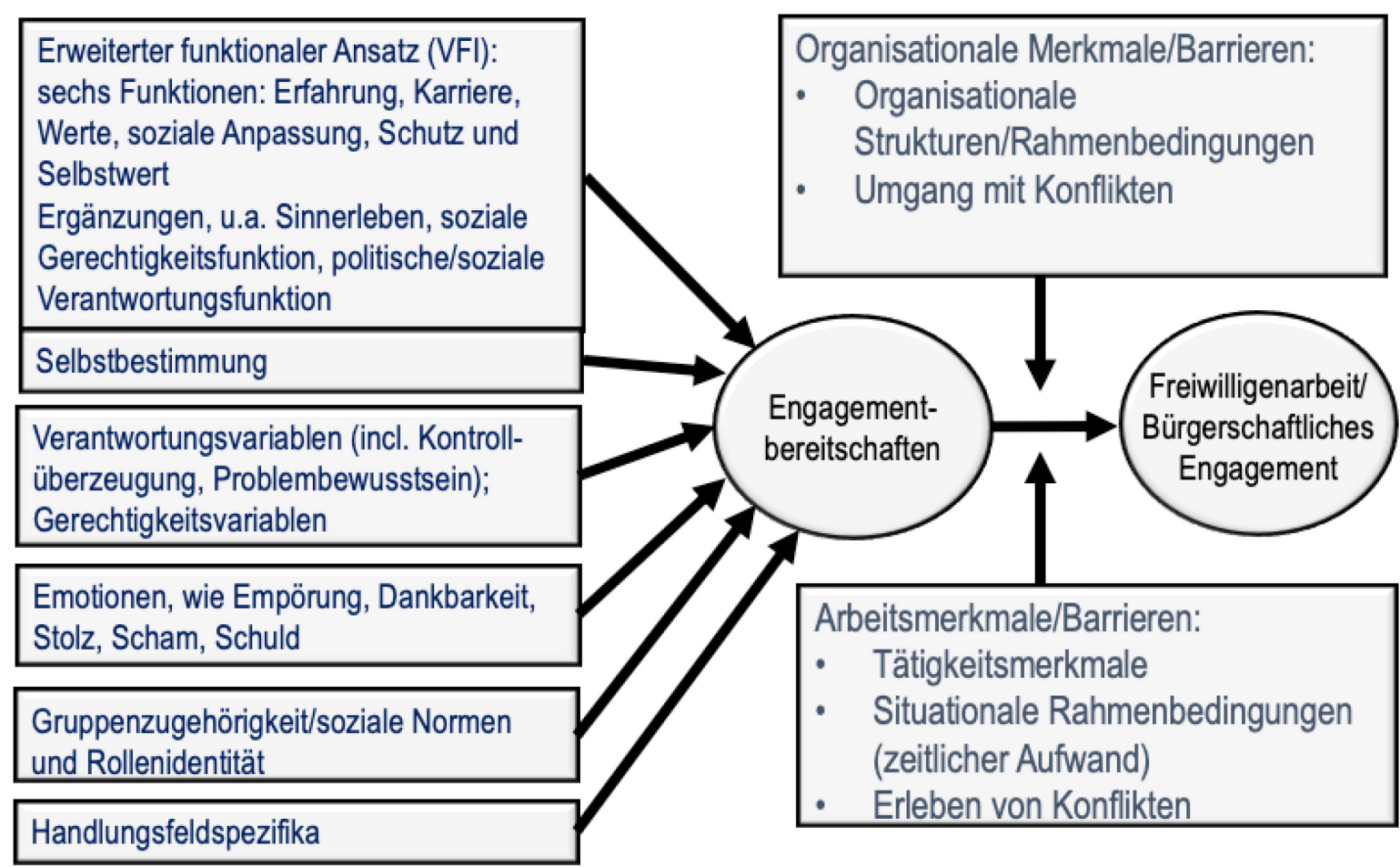

Abb. 1: Multifaktorielles Modell zur Erklärung von Freiwilligenarbeit 
Preprints 2021 der Zeitschrift BIBLIOTHEK - Forschung und Praxis, BFP.2020.0103 Kals, Strubel und Güntert

Dies ist ein Preprint $(\boldsymbol{c c})$ EY-NG-ND. Die endgültige Publikationsfassung erscheint beim Verlag De Gruyter unter https://www.degruyter.com/view/j/bfup

Als motivationale Grundlagen dieser Engagementkriterien werden fünf Gruppen von Variablen betrachtet: (1) Funktionen des Engagements nach Clary et al. ${ }^{13}$ inklusive Erweiterungen und Bezügen zur Selbstbestimmungstheorie, (2) Variablen der Kontrolle, Verantwortung und Gerechtigkeit, (3) Emotionen, (4) Identifikation, Gruppenzugehörigkeit, soziale Normen und Rollenidentität sowie (5) Variablen, die den Handlungsfeldspezifika Rechnung tragen.

Die Umsetzung der Bereitschaften in manifestes Handeln wird zudem durch Organisations- und Arbeitsmerkmale moderiert. Diese Merkmale können auch direkte Wirkung auf die Bereitschaftsbildung haben, wenn diese auf Engagements in spezifischen Organisationen ausgerichtet sind. Das Modell ist genau wie der funktionale Ansatz von Clary et al. - offen für Erweiterungen.

Die Variablen des Modells wurden über eine Vielzahl von Handlungsfeldern und durch Verfolgung sehr unterschiedlicher Forschungsansätze und -methoden validiert. Die Handlungsfelder decken sämtliche Bereiche ab, die in den Freiwilligensurveys genannt sind, differenzieren diese und grenzen sie zur bezahlten Arbeit ab. ${ }^{14}$

Die Methoden umfassen quantitative Methoden (wie Online-Befragungen mit Fragebogen), qualitative Methoden (wie halbstrukturierte Interviews) und experimentelle Ansätze. In mixed method-Ansätzen wurden zudem die verschiedenen Methodenansätze integriert und dadurch die Vorteile der unterschiedlichen Ansätze in Synergie miteinander gebracht. ${ }^{15}$

\section{Der funktionale Ansatz von Clary et al.}

Der funktionale Ansatz von Gil Clary und Kollegen bildet den Ausgangspunkt sehr vieler Arbeiten zum bürgerschaftlichen Engagement, da er innerhalb der deutschen Freiwilligenforschung aber auch auf internationaler Ebene eine hohe Bedeutung gewonnen hat. Er geht von der Annahme aus, dass eine bestimmte Freiwilligentätigkeit zugleich verschiedene individuelle unterschiedliche Funktionen für den freiwillig Tätigen erfüllen kann. Diese Funktionen sind z. T. eher selbstbezogen, z. T. stärker auf die Gemeinschaft gerichtet und lassen sich z. T. diesbezüglich nicht klar einordnen. Somit wird durch diese Funktionen die Dichotomisierung von „Altruismus“ versus „Egoismus“ überwunden, die diese Forschung zur Freiwilligenarbeit zunächst angeregt und befördert hatte.

Clary und Kollegen differenzieren sechs Funktionen von Freiwilligenarbeit, die einander in unterschiedlicher individueller Gewichtung ergänzen:

\footnotetext{
${ }^{13}$ Clary et al. (1998).

${ }^{14}$ Strubel und Kals $(2016,2018)$, Wehner und Güntert (2015).

${ }^{15}$ Freund (2020), Strubel (2019), Thiel (in Druck).
} 
(1) Erfahrungsfunktion (durch das Engagement werden praktische Erfahrungen gesammelt und Neues gelernt),

(2) Karrierefunktion (es können Fähigkeiten und Kontakte für die eigene berufliche Karriere außerhalb des Engagements erworben werden),

(3) Wertefunktion (durch das Engagement lassen sich eigene Wertvorstellungen ausdrücken und umsetzen, wie beispielsweise, anderen zu helfen),

(4) soziale Anpassungsfunktion (der Engagierte ist in eine Gruppe eingebunden und Personen, die dem Engagierten nahestehen, messen dem Engagement einen hohen Stellenwert zu),

(5) Schutzfunktion (das Engagement entlastet von Schuldgefühlen und lenkt von eigenen Sorgen $a b)$,

(6) Selbstwertfunktion (durch das Engagement wird das Selbstwertgefühl des Engagierten gesteigert).

Die Annahme dieser Funktionen wird durch viele Forschungsarbeiten gestützt. ${ }^{16}$ Zwar spielen alle Funktionen empirisch eine bedeutsame Rolle, doch von ganz besonders hoher Bedeutung sind dabei die Erfahrungs- und Wertefunktion. ${ }^{17}$

Es sind genau jene beiden Motive, die sich auch bei der Anwendung der Selbstbestimmungstheorie von Ryan und Deci ${ }^{18}$ auf freiwillige Engagements durch die Schweizer Arbeitsgruppe von den anderen Motiven unterscheiden. In dieser Theorie stehen persönliches Interesse und das Streben nach selbstbestimmtem Handeln im Vordergrund. Es zeigt sich, dass vor allem intrinsische Motivation, freiwillige Engagements befördert. ${ }^{19}$ Extrinsische Motivation, z.B. in Form finanzieller Anreize, die dazu dienen, den eigenen Lebensunterhalt zu sichern, spielen dabei keine Rolle.

Güntert ${ }^{20}$ hat die Zusammenhänge zwischen den sechs Dimensionen des funktionalen Ansatzes und den folgenden vier Motivationsarten untersucht: intrinsische Motivation (Beispiel: Die Tätigkeit macht dem Engagierten Spaß.), identifizierte Regulation (Beispiel: Die Tätigkeit findet der Engagierte persönlich wichtig.), introjizierte Regulation (Beispiel: Ohne die Tätigkeit würde sich der Engagierte schlecht fühlen.) und externale Regulation (Beispiel: Die Tätigkeit dient dazu, andere nicht zu enttäuschen.). Alle Motivationsarten hängen positiv mit allen sechs Funktionen zusammen. Es zeigt sich jedoch, dass es dabei spezifische, sinnvoll zu interpretierende Zusammenhangsmuster gibt. So hängen die Werte- als auch die Erfahrungsfunktion deutlich stärker mit selbstbestimmter als mit kontrollierter Motivation zusammen.

\footnotetext{
${ }^{16}$ Bierhoff et al. (2007), Güntert et al. (2014), Strubel und Kals (2016), Wehner und Güntert (2015).

${ }^{17}$ Strubel und Kals (2016).

${ }^{18}$ Ryan und Deci (2000).

${ }^{19}$ Güntert et al. (2016).

${ }^{20}$ Güntert (2015).
} 
Preprints 2021 der Zeitschrift BIBLIOTHEK - Forschung und Praxis, BFP.2020.0103 Kals, Strubel und Güntert

Dies ist ein Preprint $(\boldsymbol{c c})$ EY-NG-ND. Die endgültige Publikationsfassung erscheint beim Verlag De Gruyter unter https://www.degruyter.com/view/j/bfup

\section{Die Erweiterung des funktionalen Ansatzes um Sinnerleben, eine Gerechtigkeits- und eine Verantwortungsfunktion}

Clary et al. ${ }^{21}$ formulieren explizit, dass die Liste von Funktionen, die bürgerschaftliche Engagements grundsätzlich erfüllen können, erweiterbar ist. Dies geschieht in zahlreichen Forschungsarbeiten. ${ }^{22}$ Hier sind die Erweiterungen des funktionalen Ansatzes um Sinnerleben, eine Gerechtigkeits- und Verantwortungsfunktion von besonderer Bedeutung. Freiwilligenarbeit wird nach dem Wunsch einer sinnvollen Tätigkeit motiviert. ${ }^{23}$ Dabei kann Sinn auch erlebt werden, indem Gerechtigkeit gefördert wird. Die Erweiterung um eine soziale Gerechtigkeitsfunktion wurde zusammen mit der Schweizer Arbeitsgruppe um Theo Wehner vorgenommen. ${ }^{24}$ Diese Gerechtigkeitsfunktion richtet sich darauf, wahrgenommene gesellschaftliche Ungerechtigkeiten durch das eigene Engagement zu verringern und Gerechtigkeit in der Gesellschaft zu fördern. Diese Erweiterung erfolgt vor dem Hintergrund der Gerechtigkeitsmotivtheorie. Das Gerechtigkeitsmotiv ist dabei ein eigenständiges Motiv, das nicht unter Eigeninteresse subsumiert werden darf ${ }^{25}$ und sich auch von Altruismus ${ }^{26}$ unterscheidet. Die Gerechtigkeitsfunktion erklärt entsprechend einen von den bereits etablierten sechs Funktionen freiwilliger Tätigkeiten unabhängigen Varianzanteil dieser Tätigkeiten. ${ }^{27}$ Die Existenz eines solchen Gerechtigkeitsmotivs und seine Handlungswirksamkeit wird auch für spontanes prosoziales Handeln bestätigt - und dies trotz der eingangs genannten Unterschiede zu den Merkmalen der Freiwilligentätigkeit. ${ }^{28}$ Dies zeigt die Generalisierbarkeit des Befundes für unterschiedliche, jedoch inhaltlich verwandte Handlungskategorien.

Die Erweiterung durch eine politische und soziale Verantwortungsfunktion geschah durch Bierhoff und Kollegen: Bürger(innen) übernehmen durch freiwillige Tätigkeit soziale und politische Verantwortung, um gesellschaftliche Bedingungen zu verbessern. Diese Annahme wird durch eine ganze Reihe von Einzelstudien bestätigt. Dabei bezieht die Dimension der politischen und sozialen Verantwortung explizit auch den Schutz vor Ungerechtigkeit ein. ${ }^{29}$

\footnotetext{
${ }^{21}$ Clary et al. (1998).

22 Strubel und Kals (2016).

${ }^{23}$ Hoof und Schnell (2009).

24 Jiranek et al. (2013).

${ }^{25}$ Montada (2002).

${ }^{26}$ Batson et al. (1995).

${ }^{27}$ Montada (2002).

${ }^{28}$ Batson et al. (1995).

${ }^{29}$ Bierhoff et al. (2007).
} 
Preprints 2021 der Zeitschrift BIBLIOTHEK - Forschung und Praxis, BFP.2020.0103 Kals, Strubel und Güntert

Dies ist ein Preprint $(\boldsymbol{c c})$ EY-NG-ND . Die endgültige Publikationsfassung erscheint beim Verlag De Gruyter unter https://www.degruyter.com/view/j/bfup

\section{Kontroll-, Verantwortungs- und Gerechtigkeitsvariablen}

Um Kontrolle, Verantwortung und Gerechtigkeit geht es auch in der nächsten Variablengruppe. So setzt die Übernahme der Verantwortung ein Problembewusstsein sowie internale Kontrolle als wahrgenommene eigene Einflussmöglichkeiten zur Verringerung des Problems voraus, wie am Beispiel ökologischer Engagements gezeigt werden konnte. ${ }^{30}$ Eigene Interessen in Form erwarteter Reduktionen ökologischer Belastungen im eigenen Wohn- und Lebensraum spielen für diese bürgerschaftlichen Umweltengagements nur dann eine Rolle, wenn es um lokal ausgerichtete Engagements geht. ${ }^{31}$ Relevant ist hingegen das Erleben von Ungerechtigkeit aufgrund zeitlicher und geographischer Verschiebungen von Umweltbelastungen, die beispielsweise zukünftige Generationen oder ganz andere geographische Regionen belasten.

Die Gerechtigkeitsvariablen sind dabei im Sinne subjektiver Gerechtigkeitsurteile und nicht im Sinne normativ-philosophischer Aussagen gefasst. Sie teilen die Grundaussage, dass das Erleben von Ungerechtigkeit zu Handeln motiviert, um auf diese Weise die erlebte Ungerechtigkeit zu verringern. ${ }^{32}$ Dies ist nicht nur der Fall, wenn das Erleben von Ungerechtigkeit die eigene Person betrifft, sondern ebenso, wenn andere betroffen sind. Die verantwortungs- und die gerechtigkeitspsychologischen Variablen erweisen sich als hochbedeutsam, um bürgerschaftliches Engagement zu erklären und vorherzusagen:

Der Einfluss sowohl von Verantwortungsübernahme als auch von Gerechtigkeitsüberzeugungen wird bereits durch die frühe Arbeit von Moschner ${ }^{33}$ gestützt. Sie untersuchte, welchen Einfluss Gerechtigkeitsüberzeugungen auf die Übernahme allgemeiner Verantwortung in politischer, gesellschaftlicher, sozialer und kultureller Hinsicht haben und wie sich diesbezüglich freiwillig Engagierten von Nicht-Engagierten unterscheiden. Es bestätigte sich, dass freiwillig Engagierte höhere Absichten zur Verantwortungsübernahme und stärkere Gerechtigkeitsüberzeugen hatten (im Sinne einer höheren Gerechtigkeitszentralität und geringerem Gerechte-Welt-Glauben). Beides konnte direkt oder indirekt auch die Freiwilligentätigkeiten erklären.

Der Einfluss des Glaubens an eine Gerechte Welt nach Lerner, ${ }^{34}$ der Überzeugung, dass es auf der Welt gerecht zugehe und jeder bekomme was er verdiene und verdiene was er bekomme, für Freiwilligenarbeit als Teildimension sozialen Aktivismus bestätigt sich schließlich auch in der Studie

\footnotetext{
${ }^{30}$ Kals et al. (2001).

${ }^{31}$ Kals et al. (2001).

32 vgl. Montada (2002).

${ }^{33}$ Moschner (1998).

34 Lerner (1980).
} 
Preprints 2021 der Zeitschrift BIBLIOTHEK - Forschung und Praxis, BFP.2020.0103 Kals, Strubel und Güntert

Dies ist ein Preprint $(\boldsymbol{c c})$ EY-NG-ND. Die endgültige Publikationsfassung erscheint beim Verlag De Gruyter unter https://www.degruyter.com/view/j/bfup

von Moore. ${ }^{35}$ Dieser Studie zufolge hemmt ein hoher Glaube an eine gerechte Welt diesen sozialen Aktivismus. Auch in anderen Studien erwies sich der Glaube an eine Gerechte Welt als wichtig, um freiwillige Engagements zu erklären. ${ }^{36}$

Neufeind und Kollegen gelang dabei eine Integration des funktionalen Ansatzes, der Gerechtigkeitsüberzeugungen (Gerechtigkeitszentralität und Glaube an eine gerechte Welt) sowie der politischen und sozialen Verantwortung nach Bierhoff et al. ${ }^{37}$ Auch hier ging es darum, Freiwilligenforschung mit der Gerechtigkeitstradition zu verbinden. Abermals erwiesen sich der funktionale Ansatz sowie die Gerechtigkeits- und Verantwortungsüberzeugungen als ergänzend bedeutsam, um freiwillige Tätigkeiten zu erklären.

Die Befunde einer komplexen Studienreihe zum nachhaltigen Konsum und damit verbundenen Engagements zur Förderung des Fairen Handelns sprechen ebenfalls dafür, dass diese sowohl durch nutzenbezogene Motive als auch durch Motive der Verantwortung und Gerechtigkeit motiviert sind. ${ }^{38}$ Hier zeigt sich damit der Motivpluralismus aber auch die rmoral- und verantwortungsbezogene Basis eines Engagements in besonderer Weise. So tragen Gerechtigkeitsurteile bedeutsam dazu bei, dass überhaupt die Notwendigkeit einer Handlung erkannt wird. Die Überzeugung etwas bewirken zu können und die Zuschreibung von Verantwortung an die eigene Person und einflussreiche Dritte sind wichtig damit tatsächlich Verantwortung übernommen wird.

\section{Emotionsvariablen}

Die Bedeutung des Gerechtigkeitsmotivs für menschliches Handeln wurde somit für zahlreiche Handlungsfelder nachgewiesen und konnte auch für freiwillige Tätigkeiten bestätigt werden. ${ }^{39}$ Dabei spielen jedoch nicht nur Gerechtigkeits- oder Verantwortungsüberzeugungen als Kognitionen eine Rolle, sondern ebenso als Emotionen, wobei Empörung der emotionale Leitindikator erlebter Ungerechtigkeit ist.

Auch darüber hinaus sind nicht nur Kognitionen als Motive wirksam, sondern ebenfalls Emotionen. ${ }^{40}$ Dazu wurden die Skalen des funktionalen Ansatzes nach Clary et al. ${ }^{41}$ um Skalen zur Erfassung der

\footnotetext{
35 Moore (2008).

${ }^{36}$ vgl. Neufeind et al. (2014).

${ }^{37}$ Bierhoff et al. (2007).

${ }^{38}$ Strubel $(2019,2020)$.

${ }^{39}$ Moore (2008); Moschner (1998); Neufeind et al. (2014).

${ }^{40}$ Thiel, Strubel und Kals (2016).

${ }^{41}$ Clary et al. (1998).
} 
Preprints 2021 der Zeitschrift BIBLIOTHEK - Forschung und Praxis, BFP.2020.0103 Kals, Strubel und Güntert

Dies ist ein Preprint $(\boldsymbol{c c})$ EY-NG-ND. Die endgültige Publikationsfassung erscheint beim Verlag De Gruyter unter https://www.degruyter.com/view/j/bfup

Emotionen der Dankbarkeit, des Stolzes, der Scham und Schuld sowie der Empörung ergänzt. Die Ergebnisse zeigen, dass sich die erstmalig erfassten emotionsbezogenen Motivatoren von anderen Motiven abgrenzen lassen und darüber hinaus einen zusätzlichen Beitrag zur Vorhersage der Intentionen zur politischen Partizipation, als einer niedrigschwelligen Form bürgerschaftlichen Engagement, leisten. Auch hier erweist sich die Empörungsfunktion als besonders bedeutsam.

\section{Identifikation, Gruppenzugehörigkeit, soziale Normen und Rollenidentität}

Die Rolle von Identifikation, Gruppenzugehörigkeit, soziale Normen und Rollenidentität wurde ebenfalls in vielen Forschungsarbeiten bestätigt. ${ }^{42}$ So werden viele bürgerschaftliche Engagements im Rahmen von Organisationen erbracht, wie Caritas, Rotes Kreuz, Amnesty International, Greenpeace, freiwillige Feuerwehr etc. Zugleich werden durch die Arbeit in einer Freiwilligenorganisation soziale Normen aktiviert, da hier viele Gleichgesinnte gemeinsam und oft auch miteinander arbeiten.

Um die Ebenen der Freiwilligenarbeit und der Organisation zusammenzubringen, haben Grube und Piliavin ${ }^{43}$ die Identitätstheorie von Stryker und Burke ${ }^{44}$ in Form einer Rollenidentitätstheorie auf die Freiwilligenarbeit angewandt. Der zufolge stehen bei freiwilligen Engagements, die in Organisationen erbracht werden, zwei Rollenidentitäten in potentieller Konkurrenz zueinander: einerseits die generelle Identität als freiwillig Tätiger und andererseits eine organisationsspezifische Identität, die die Arbeit speziell in der einen Organisation betrifft, in der man freiwillig tätig ist. Dabei können beide Identitäten und damit die Absicht, sich für das jeweilige Ziel oder für eine bestimmte Organisation einzusetzen, zusammenfallen. Dies sollte vor allem bei Organisationen der Fall sein, die für die jeweilige Erreichung des Ziels Alleinstellungsmerkmale besitzen, wie Amnesty International. ${ }^{45}$ Wenn sich jedoch unterschiedliche Organisationen für dasselbe Zielspektrum engagieren und sich somit Wahlmöglichkeiten für freiwillig Engagierte stellen, in welcher Organisation sie ihr Engagement einbringen wollen, stehen die Organisationen miteinander in einem Wettbewerb um die freiwillig Tätigen. Gleichzeitig gewinnt die organisationsspezifische Identität der freiwillig Tätigen an Bedeutung, da z.B. ein Wechsel in eine andere Organisation mit gleicher Zielausrichtung möglich wäre.

\footnotetext{
42 Freund (2020); Thiel (in Druck); van Schie et al. (2014).

${ }^{43}$ Grube und Piliavin (2000).

${ }^{44}$ Stryker und Burke (2000).

${ }^{45}$ Vgl. Wehner und Güntert (2015).
} 
Preprints 2021 der Zeitschrift BIBLIOTHEK - Forschung und Praxis, BFP.2020.0103 Kals, Strubel und Güntert

Dies ist ein Preprint $(\boldsymbol{c c})$ EY-NG-ND . Die endgültige Publikationsfassung erscheint beim Verlag De Gruyter unter https://www.degruyter.com/view/j/bfup

Entsprechend hat auch Penner ${ }^{46}$ die Rollenidentitätstheorie mit dem funktionalen Ansatz in ein gemeinsames Modell integriert. Die zentrale Aussage ist, dass der funktionale Ansatz vor allem erklärt, warum jemand eine Freiwilligentätigkeit aufgreift, während die Rollenidentitätstheorie vor allem vorhersagt, warum jemand bei dieser Tätigkeit in der jeweiligen Organisation bleibt oder aber wechselt bzw. ganz abbricht. Die Bildung einer organisationsspezifischen Identität ist hierfür zentral und kann von einer generellen Identität als freiwillig Tätiger empirisch unterschieden werden. ${ }^{47}$

\section{Handlungsfeldspezifische Motivvariablen}

Die organisationsspezifische Identität weist bereits darauf hin, dass es Variablen und Motive gibt, die für das jeweilige Handlungsfeld spezifisch sind. Diese spezifischen Motivvariablen konnten wir für sehr viele Handlungsfelder nachweisen. Dazu zwei Beispiele:

1. Beim bürgerschaftlichen Engagement im Kontext der Flüchtlingshilfe spielen Fragen der Gerechtigkeit und wie inklusiv diese gedacht wird, eine besondere Rolle. ${ }^{48}$ Dies drückt sich im "Scope of Justice" aus, der besagt, inwiefern die eigenen Gerechtigkeitsvorstellungen auch für Flüchtlinge gelten. Er erklärt gemeinsam mit Empörung über zu wenig Flüchtlingshilfe und abgelehnter Empörung über zu viel Flüchtlingshilfe 63 Prozent der Unterschiedlichkeit in den Bereitschaften zu Engagements in der Flüchtlingshilfe. Der Scope of Justice Nichtengagierter ist zudem geringer ausgeprägt als der einer engagierten Vergleichsgruppe.

2. Bei der Freiwilligen Feuerwehr sowie der Caritas erweisen sich Wertorientierungen als besonders wichtig, um die Bildung von Engagements sowie Bereitschaften zur Unterstützung notwendiger Transformationen der Organisationen zu erklären. ${ }^{49}$ Darüber hinaus ist bei der Freiwilligen Feuerwehr auch das Motiv des Sensation Seeking, als Suche nach Abenteuer und aufregendem Erleben von Bedeutung. ${ }^{50}$

Auch handlungsfeldspezifische Erweiterungen des funktionalen Ansatzes haben sich als erfolgreich erwiesen. ${ }^{51}$

\section{Moderierende situationale und organisationspsychologische Faktoren}

In einer frühen Studie zur Bildung von Engagements im Bereich des Umweltschutzes konnte im Längsschnitt gezeigt werden, dass grundsätzliche Bereitschaften valide Prädiktoren tatsächlichen Engagements sind. ${ }^{52}$ Allerdings wurde die Umsetzung solcher Bereitschaften durch die jeweiligen Rahmenbedingungen moderiert. Dies bestätigt sich auch für freiwillige Engagements. Sind die Engagements in eine Organisation eingebunden, so wirken vor allem starre organisationale

\footnotetext{
46 Penner (2002).

${ }^{47}$ van Schie et al. (2014).

48 Strubel und Kals (2018).

${ }^{49}$ Freund (2020).

50 Kals et al. (2016).

${ }^{51}$ Güntert et al. (2014), Strubel und Kals (2016).

${ }^{52}$ Montada et al. (2007).
} 
Preprints 2021 der Zeitschrift BIBLIOTHEK - Forschung und Praxis, BFP.2020.0103 Kals, Strubel und Güntert

Dies ist ein Preprint $(\boldsymbol{c c})$ EY-NG-ND. Die endgültige Publikationsfassung erscheint beim Verlag De Gruyter unter https://www.degruyter.com/view/j/bfup

Strukturen als Barrieren für die Umsetzung der Bereitschaften oder führen dazu, dass Engagement wieder aufgegeben wird. ${ }^{53} \mathrm{Zu}$ den situationalen Rahmenbedingungen gehört zudem die Machbarkeit der zeitlichen Anforderungen. So sind zwar viele Bürgerinnen und Bürger auch zu zeitlich umfänglichen Engagements bereit, doch wirkt der Zeitaufwand als starke Barriere, wenn nicht genügend zeitliche Flexibilität zum Einbringen des Engagements bereitgestellt wird. Dies geht Hand in Hand mit starren organisationalen Strukturen. Dann sind die Engagements beispielsweise nur schwer mit der beruflichen Tätigkeit in Einklang zu bringen. ${ }^{54}$

\section{Das Erleben organisationsinterner Konflikte}

Eine weitere große Barriere bildet das Erleben von organisationsinternen Konflikten bei der Ausführung freiwilliger Engagements. Diese Konflikte können dazu führen, dass trotz grundsätzlicher Bereitschaften die Engagements abgebrochen werden. Dies vor allem, da die Engagements ohnehin freiwillig und unbezahlt erfolgen, wodurch keine wirtschaftliche Abhängigkeit gegeben ist. ${ }^{55}$

Die erlebten Konflikte lassen sich dabei in der großen Mehrzahl als Gerechtigkeitskonflikte verstehen, bei denen soziale Normen und Ansprüche als verletzt wahrgenommen werden. Diese Konflikte basieren auf spezifischen gerechtigkeitsbezogenen Urteilen. Gerechtigkeitskonflikte spielen im Bereich der Freiwilligenarbeit durchaus eine bedeutsame Rolle, denn Gerechtigkeit ist, wie beschrieben, ein wichtiges Motiv zur Aufnahme eines Engagements. ${ }^{56}$ Zudem ist Gerechtigkeitszentralität in der Stichprobe der freiwillig Engagierten hoch ausgeprägt. ${ }^{57}$ Wird Ungerechtigkeit in der Arbeit erfahren oder werden gerechtigkeitsbezogene Erwartungen an die Tätigkeit selbst nicht erfüllt, so könnte dies sensibel wahrgenommen werden und zu latenten oder manifesten Konflikten führen.

Bis vor Kurzem gab es zu diesen Überlegungen noch so gut wie keine empirischen Daten. Daher hat eine neue Studienreihe besonderes Gewicht, in der das Erleben von Konflikten im bürgerschaftlichen Engagement bzw. im Ehrenamt empirisch untersucht wird. ${ }^{58}$ So konnte an einer Stichprobe von Freiwilligen unterschiedlicher Organisationen die Bedeutung von Konflikten und hier vor allem die Bedeutung des Umgangs mit ihnen gezeigt werden. Analog zu Befunden in Profit-Organisationen bilden sich Beziehungs-, Beurteilungs- sowie Prozesskonflikte als drei Typen von Konflikten ab.

\footnotetext{
${ }^{53}$ Freund (2020).

${ }^{54}$ van Schie et al. (2015), Thiel (in Druck).

55 Kals et al. (2019); Thiel (in Druck).

56 Jiranek et al. (2013).

57 Neufeind et al. (2014).

58 Thiel (in Druck).
} 
Preprints 2021 der Zeitschrift BIBLIOTHEK - Forschung und Praxis, BFP.2020.0103 Kals, Strubel und Güntert

Dies ist ein Preprint $(\boldsymbol{c c})$ EY-NG-ND. Die endgültige Publikationsfassung erscheint beim Verlag De Gruyter unter https://www.degruyter.com/view/j/bfup

Beziehungs- und Prozesskonflikte bilden dabei wichtige Einflussgrößen für das Erleben von Unzufriedenheit sowie für die Rückzugsabsicht. Dies steht in Einklang mit früheren Erkenntnissen, dass das Erleben von Gerechtigkeitskonflikten in Zusammenhang mit der Beendigung von Engagements und Mitgliederschwund steht. ${ }^{59}$ Entscheidend ist, wie intensiv und häufig die Konflikte wahrgenommen werden und - damit zusammenhängend - wie in der Organisation bzw. in dem sozialen Miteinander mit ihnen umgegangen wird.

Die Studie von Thiel steht aber auch in Einklang mit dem Befund, dass der Verfahrensgerechtigkeit in der Bearbeitung von Konflikten in Gruppen und Organisationen eine bedeutende Rolle zukommt. ${ }^{60}$ Eine wichtige Wirkung von Verfahrensgerechtigkeit wird im Group Engagement-Modell von Tyler und Blader ${ }^{61}$ beschrieben. Hier wird angenommen, dass sich Verfahrensgerechtigkeit in Gruppen vermittelt über die soziale Identität auf Variablen der Kooperation auswirkt und daher besonders bedeutsam ist. Dieses Modell hat sich im Kontext von Profit-Organisationen bewährt. Anhand einer internationalen Fragebogenstudie an Freiwilligen zeigt sich, dass es sich auch auf organisierte Freiwilligenarbeit übertragen lässt. Daher ist Verfahrensgerechtigkeit bei der Bearbeitung von Konflikten im Freiwilligensektor umfänglich zu berücksichtigen.

\section{Moderierende arbeitspsychologische Faktoren}

Auch die Merkmale der Tätigkeit selbst sind von hoher Bedeutung. Bei der Untersuchung der Tätigkeitsmerkmale wird auf den großen Wissensfundus im Bereich der bezahlten Arbeit zurückgegriffen, hier u.a. auf das etablierte Job-Characteristics Modell von Hackman und Oldham. ${ }^{62}$ Dieses Modell umfasst fünf Kernmerkmale der Arbeit: Variabilität, Ganzheitlichkeit, Bedeutung, Autonomie und Feedback, die u.a. das Erleben von Sinnhaftigkeit und Verantwortlichkeit als Erlebniszustände erklären, die sich zugleich als wichtige Motivatoren für bürgerschaftliches Engagement erwiesen haben. Die Erlebniszustände der Sinnhaftigkeit und Verantwortlichkeit haben zugleich Auswirkungen auf die Förderung intrinsischer Motivation, die Arbeitszufriedenheit sowie verschiedene Leistungskriterien.

Dieses Modell konnte erfolgreich auf Freiwilligenarbeit angewandt werden. ${ }^{63}$ Als besonders wichtig haben sich die Kernmerkmale der Variabilität und der Bedeutung der Arbeit sowie des direkten

\footnotetext{
${ }^{59}$ Gensicke und Geiss (2009).

${ }^{60}$ Strubel und Kals (2017).

61 Tyler und Blader (2003).

62 Hackman und Oldham (1975).

${ }^{63}$ Thiel (in Druck).
} 
Preprints 2021 der Zeitschrift BIBLIOTHEK - Forschung und Praxis, BFP.2020.0103 Kals, Strubel und Güntert

Dies ist ein Preprint $(\boldsymbol{c c})$ EY-NG-ND. Die endgültige Publikationsfassung erscheint beim Verlag De Gruyter unter https://www.degruyter.com/view/j/bfup

Feedbacks durch Vorgesetzte erwiesen. ${ }^{64}$ Der Einfluss von Bedeutung und Feedback spiegelt sich auch in dem Befund, dass eine unzureichende Wertschätzung des Engagements in der allgemeinen Bevölkerung, beim Arbeitgeber und in der Politik dazu führt, dass ein Engagement aufgegeben oder gar nicht erst aufgenommen wird. ${ }^{65}$

\section{Zusammenfassung und Ableitungen für die Förderung von Freiwilligenarbeit sowie die weitere Forschung}

In der Gesamtschau haben wir viel Wissen über Bereitschaften zu Freiwilligenarbeit in der allgemeinen Bevölkerung und damit über potentiell Engagierte. Zudem haben wir bereits gesichertes Wissen über die grundlegenden Motivlagen für Freiwilligenarbeit in unterschiedlichen Feldern und innerhalb der Gruppe von Engagierten, wie der Freiwilligen Feuerwehr, dem Katastrophenschutz, dem Engagement in einem großen katholischen Sozialverband. ${ }^{66}$ Die wesentlichen Antworten auf die Frage, aufgrund welcher Motivlagen Bürgerinnen und Bürger die Bereitschaft bilden, sich freiwillig zu engagieren, das Engagement aufnehmen und es auch über die Zeit fortsetzen, lassen sich wie folgt zusammenfassen:

- Die Motivlagen sind multifaktoriell und umfassen vielfältige Motive, die in unterschiedlichen Anteilen sowohl selbstbezogene als auch auf das Gemeinwohl bezogene und damit altruistische Motive umfassen. Damit bestätigt sich auch in diesem Forschungsfeld, dass das Menschenbild des homo oeconomicus zu kurz greift.

- Ausgehend von der funktionalen Vielfalt, die Freiwilligenarbeit in der Ursprungsfassung des Ansatzes von Clary et al. ${ }^{67}$ erklärt, sind alle sechs Funktionen von Relevanz. Doch besonders hohe Bedeutung haben empirisch die Werte- und Erfahrungsfunktion erlangt. Es sind auch genau diese beiden Funktionen, also die Werte- als auch die Erfahrungsfunktion, die im Sinne der Selbstbestimmungstheorie deutlich stärker mit selbstbestimmter als mit kontrollierter Motivation zusammenhängen.

- Über diesen Ursprungsansatz hinaus haben sich die ergänzten Motive bzw. Funktionen des Erlebens von Sinn, von Gerechtigkeit sowie von politischer oder sozialer Verantwortung ebenfalls als besonders bedeutsam erwiesen.

- Entsprechend wurden die zentralen Verantwortungs- und Gerechtigkeitsvariablen weiter ausdifferenziert. Der Übernahme von Verantwortung geht dabei voraus, dass ein Problembewusstsein für das gesellschaftliche Thema (wie Flüchtlingshilfe) besteht, in der das Engagement erbracht wird. Dieses Engagement muss darüber hinaus als wirkungsvoll im Sinne einer internalen Kontrollüberzeugung erlebt werden.

- Die Motive bilden sich gleichermaßen als kognitive Bewertungen und Urteile wie auch als Emotionen ab. Emotionen, wie Empörung über als ungerecht erlebte Verteilungen oder Zustände, sind dabei keine „Epi-Phänomene" der Kognitionen, sondern haben in gleicher Weise wie die Kognitionen eigenständige Erklärungskraft.

\footnotetext{
${ }^{64}$ Güntert (2014); Thiel (in Druck); van Schie et al. (2014).

${ }^{65}$ Freund (2020); Thiel (in Druck).

${ }^{66}$ Freund (2020); Strubel (2019); Thiel (in Druck).

${ }^{67}$ Clary et al. (1998).
} 
Dies ist ein Preprint $(\boldsymbol{c c}) \mathbf{E Y}$-NG-ND. Die endgültige Publikationsfassung erscheint beim Verlag De Gruyter unter https://www.degruyter.com/view/j/bfup

- Identifikation, Gruppenzugehörigkeit und Rollenidentität erklären nicht nur, warum sich jemand bürgerschaftlich engagiert, sondern auch, warum er oder sie sich genau in diesem spezifischen Feld oder in einer ausgewählten Freiwilligenorganisation engagiert. Das Erleben von Zugehörigkeit und Gemeinschaft ist dabei der psychologische Kern. ${ }^{68}$

- Schließlich gibt es spezifische Motive, die dazu führen, dass sich jemand speziell in einem Handlungsfeld engagiert, wie das Sensation Seeking als Motiv für ein Engagement in der Freiwilligen Feuerwehr. ${ }^{69}$

- Organisationale und Tätigkeitsmerkmale erklären, was den Transfer grundlegender Bereitschaften in manifestes Handeln befördert aber auch hemmt. Hierzu gehören starre organisationale Strukturen, ein dysfunktionaler Umgang mit Konflikten aber auch Tätigkeitsmerkmale, die das Erleben von Sinn, erlebter Verantwortung und Ganzheitlichkeit hemmen, sowie ein (zu) hoher zeitlicher Aufwand, der mit dem Engagement einhergeht. Welche praktischen Schlussfolgerungen lassen sich nun aus den Befunden zur Gewinnung neuer Engagierter und zur langfristigen Bindung bereits Engagierter ziehen?

Aus allen beschriebenen empirischen Befunden leiten sich hierzu direkte Empfehlungen ab:

- Werbe-, Image- und Aufklärungskampagnen und -strategien sollten an diesen Motiven ansetzen und zeigen, wie sie sich durch freiwillige Engagements erfüllen lassen.

- Erkenntnisse zu den Organisations- und Arbeitsmerkmalen sollten genutzt werden, um eine möglichst hohe Erfüllung der Motive sowie eine hohe Passung zwischen Erwartungen und Anforderungen zu erreichen. Das betrifft den Wunsch nach Erfüllung der Motive, nach Verbundenheit und nach Rollenidentität gleichermaßen. ${ }^{70}$

- Die arbeits- und organisationspsychologischen Bedingungen des bürgerschaftlichen Engagements sind genauso sorgfältig zu gestalten, wie dies auch für bezahlte Tätigkeiten geschieht. Alle genannten Kernmerkmale der Arbeit sind in positivem Maße zu erfüllen. Dies gilt in besonderer Weise für die Merkmale „Variabilität", „Bedeutung der Arbeit“ sowie "direktes Feedback", denn diese haben sich als ganz besonders wichtig erwiesen.

Der Befund, dass es organisations- bzw. handlungsfeldspezifische Identifikationen und

Rollenidentitäten gibt, bestätigt, dass es nicht eine homogene Gruppe „freiwillig Engagierter" gibt, sondern, dass spezifische Interessen und Motivlagen existieren, die es differentiell zu berücksichtigen gilt. Ziel ist immer einen möglichst hohen Person-Environment-Fit zu erreichen. Dies gilt in der bezahlten wie in der unbezahlten Arbeit gleichermaßen.

Neben der Motiverfüllung, den Organisations- und Arbeitsmerkmalen ist dem Umgang mit Konflikten bei den bürgerschaftlichen Engagements besondere Aufmerksamkeit zu schenken. Konflikte sind in diesem Handlungsfeld oftmals tabuisiert und trotzdem gehören sie immer dann zum Alltag, wenn Menschen miteinander arbeiten und tätig sind. Basierend auf den vorgestellten Befunden, ${ }^{71}$ finden sich in der Literatur wissenschaftlich fundierte Praxisempfehlungen, wie mit diesen Konflikten im Selbstmanagement oder auch unter Hinzunahme eines Konfliktberaters psychologisch fundiert

\footnotetext{
${ }^{68}$ Kals et al. (2019); Strubel und Kals (2016).

${ }^{69}$ Kals et al. (2016).

${ }^{70}$ van Schie et al. (2015).

${ }^{71}$ Thiel (in Druck).
} 
Preprints 2021 der Zeitschrift BIBLIOTHEK - Forschung und Praxis, BFP.2020.0103 Kals, Strubel und Güntert

Dies ist ein Preprint $(\boldsymbol{c c})$ EY-NG-ND . Die endgültige Publikationsfassung erscheint beim Verlag De Gruyter unter https://www.degruyter.com/view/j/bfup

umgegangen werden kann, um keine Engagierten zu verlieren, sondern den Konflikt konstruktiv zu lösen und als gemeinsame Entwicklungschance zu nutzen. ${ }^{72}$

Eingrenzungen von Freiheit und Autonomie durch starre Vorgaben oder Strukturen, die

Wahrnehmung, dass freiwillige Engagements durch Bezahlung gelenkt und möglicherweise sogar instrumentalisiert werden, aber auch mangelnde Wertschätzung oder das Erleben von Konflikten können Engagierte dazu bringen, dass sie ihr Engagement aufgeben. Positives Feedback und Wertschätzung der Engagements sind hingegen wichtige Antriebsquelle und Motivation für die Engagierten. Genau diese Wertschätzung erleben viele Engagierte aber auch viele Freiwilligenorganisationen in Krisenzeiten, wie etwa in der Corona-Krise, in besonderer Weise. Angesichts der Bedeutung, die freiwillige Engagements für die Zivilgesellschaft haben, ist diese Wertschätzung wohlverdient und fördert das Erleben von Gemeinschaft.

\section{Literaturverzeichnis}

Batson, C. D., Klein, T. R., Highberger, L. \& Shaw, L. L. (1995). Immorality from empathy induced altruism: When compassion and justice conflict. Journal of Personality and Social Psychology, 68, 1042-1054.

Bierhoff, H.-W., Schülken, T. \& Hoof, M. (2007). SEEH - Skalen der Einstellungsstruktur ehrenamtlicher Helfer. Zeitschrift für Personalpsychologie, 6, 12-27.

Clary, E. G., Snyder, M., Ridge, R. D., Copeland, J., Stukas, A. A., Haugen, J., \& Miene, P. (1998). Understanding and assessing the motivations of volunteers: A functional approach. Journal of Personality and Social Psychology, 74, 1516-1530.

Dovidio, J. F., Piliavin, J. A., Schroeder, D. A. \& Penner, L. A. (2006). The social psychology of prosocial behavior. Mahwah, NJ: Lawrence Erlbaum.

Freund, S. (2020). Organisationsentwicklung in Freiwilligenorganisationen: psychologische Modellbildung und Evaluation von Veränderungsbereitschaft und Engagement im Transformationsprozess. Wiesbaden: Springer.

Gensicke, T. \& Geiss, S. (2009). Hauptbericht des Freiwilligensurveys 2009: Ergebnisse der repräsentativen Trenderhebung zu Ehrenamt, Freiwilligenarbeit und Bürgerschaftlichem Engagement: Zusammenfassung. München: Bundesministerium für Familie, Senioren, Frauen und Jugend.

Gollwitzer, M., Lotz, S., Schlösser, T. \& Streicher, B. (Hrsg.). (2013). Soziale Gerechtigkeit. Göttingen: Hogrefe.

Grube, J. A. \& Piliavin, J. A. (2000). Role identity, organizational experiences, and volunteer performance. Personality and Social Psychology Bulletin, 26, 1108-1119.

Güntert, S. T. (2014). The impact of work design, autonomy support, and strategy on employee outcomes: A differentiated perspective on self-determination at work. Motivation and Emotion, 39(1), 74-87.

\footnotetext{
72 Kals et al. (2019).
} 
Preprints 2021 der Zeitschrift BIBLIOTHEK - Forschung und Praxis, BFP.2020.0103 Kals, Strubel und Güntert

Dies ist ein Preprint $(\boldsymbol{c c})$ EY-NG-ND . Die endgültige Publikationsfassung erscheint beim Verlag De Gruyter unter https://www.degruyter.com/view/j/bfup

Güntert, S. T. (2015). Selbstbestimmung in der Freiwilligenarbeit. In T. Wehner \& S. T. Güntert (Hrsg.), Psychologie der Freiwilligenarbeit. Motivation, Gestaltung und Organisation (S. 77-93). Berlin: Springer.

Güntert, S. T., Neufeind, M., \& Wehner, T. (2014). Motives for event volunteering: Extending the functional approach. Nonprofit and Voluntary Sector Quarterly. Advance online publication.

Güntert, S.T., Strubel, I. T., Kals, E. \& Wehner, T. (2016). The quality of volunteers' motives: Integrating the functional approach and self-determination theory. Journal of Social Psychology, 156(3), 310-327.

Hackman, J.R. \& Oldham, G.R. (1975). Development of the job diagnostic survey, Journal of Applied Psychology 60 (2), 159-170.

Hoof, M., \& Schnell, T. (2009). Sinn-volles Engagement: Zur Sinnfindung im Kontext der Freiwilligenarbeit. Wege zum Menschen, 61(5), 405-422.

Jiranek, P., Kals, E., Humm, J. S., Strubel, I. T., \& Wehner, T. (2013). Volunteering as a means to an equal end? The impact of a social justice function on intention to volunteer. Journal of Social Psychology, 153, 520-541.

Kals, E. (1999). Der Mensch nur ein zweckrationaler Entscheider? Zeitschrift für Politische Psychologie, 3, 267-293.

Kals, E., Maes, J. \& Becker, R. (2001). The overestimated impact of self-interest and the underestimated impact of justice motives. Trames. Journal of Humanities and Social Sciences, 55 (3), 269-287.

Kals, E., Strubel, I. T., Vaganian, L., Güntert, S.T. \& Wehner, T. (2016). Freiwilligenarbeit und Erwerbsarbeit am Beispiel der Feuerwehr: Mehr Gemeinsamkeiten als Unterschiede. Wirtschaftspsychologie, 2, 67-79.

Kals, E., Thiel, K. \& Freund, S. (2019). Praxishandbuch zur Lösung von Konflikten im Ehrenamt. Stuttgart: Kohlhammer.

Lerner, M. J. (1980). The belief in a just world. A fundamental delusion. New York: Plenum Press.

Miller, M. (1994). Ellbogenmentalität und ihre theoretische Apotheose: Einige kritische Anmerkungen zur Rational Choice Theory. Soziale Welt, 45(1), 5-15.

Montada, L. (2002). Doing justice to the justice motive. In M. Ross \& D. T. Miller (Eds.), The justice motive in everyday life (pp. 41-62). Cambridge, UK: Cambridge University Press.

Montada, L., Kals, E. \& Becker, R. (2007). Willingness for continued social commitment: A new concept in environmental research. Environment \& Behavior, 39 (3), 287-316.

Moore, D. (2008). Toward a more just world: What makes people participate in social action? In K. A. Hegvedt \& J. Clay-Warner (Eds.), Advances in group processes (Vol. 25: Justice) (pp. 213-239). Bingley: Emerald Group Publishing.

Moschner, B. (1998). Ehrenamtliches Engagement und soziale Verantwortung. In B. Reichle \& M. Schmitt (Hrsg.), Verantwortung, Gerechtigkeit und Moral. Zum psychologischen Verständnis ethischer Aspekte im menschlichen Verhalten (S. 73-86). Weinheim: Juventa.

Neufeind, M., Jiranek, P. \& Wehner, T. (2014). Beyond skills and structure: Justice dispositions as antecedents of young citizens' volunteering and political participation. Journal of Community \& Applied Social Psychology. 24 (4), 278-295.

Penner, L. A. (2002). Dispositional and organizational influences on sustained volunteerism: An interactionist perspective. Journal of Social Issues, 58 (3),447-467. 
Preprints 2021 der Zeitschrift BIBLIOTHEK - Forschung und Praxis, BFP.2020.0103 Kals, Strubel und Güntert

Dies ist ein Preprint $(\boldsymbol{c c})$ EY-NG-ND . Die endgültige Publikationsfassung erscheint beim Verlag De Gruyter unter https://www.degruyter.com/view/j/bfup

Röbke, R. (2012). Bürgerschaftliches Engagement und sozialstaatliche Daseinsvorsorge. Arbeitskreis Bürgergesellschaft und aktivierender Staat. Berlin: Friedrich-Ebert-Stiftung. https://library.fes.de/pdf-files/do/08956.pdf

Ryan, R. M., \& Deci, E. L. (2000). Self-determination theory and the facilitation of intrinsic motivation, social development, and well-being. American psychologist, 55(1), 68.

Simonson, J., Vogel, C. \& Tesch-Römer, C. (2016). Freiwilliges Engagement in Deutschland. Der Deutsche Freiwilligensurvey 2014. Berlin: Bundesministerium für Familie, Senioren, Frauen und Jugend.

Strubel, I. T. (2020). The impact of indignation on Fairtrade support. Conflict \& Communication online, 19(1\&2).

Strubel, I. T. (2019). Nachhaltiger Konsum, Fairer Handel und Gerechtigkeit: eine multimethodale psychologische Untersuchung gerechtigkeits- und verantwortungsbezogener Motive. Katholische Universität Eichstätt-Ingolstadt: Dissertation. Online: https://opus4.kobv.de/opus4-kueichstaett/frontdoor/index/index/docld/496

Strubel, I. T. \& Kals, E. (Hrsg.). (2016). Freiwilligenarbeit und Gerechtigkeit. Zürich: Zentrum für Organisations- und Arbeitswissenschaften, ETH Zürich.

Strubel, I. T. \& Kals. E. (2017). Verfahrensgerechtigkeit zählt: eine Übertragung des Group Engagement Modells auf Freiwilligenarbeit im Kontext von Organisationen. Konfliktdynamik 2(6),128-135.

Strubel, I. T. \& Kals, E. (2018). Scope of Justice und freiwillige Engagements in der Flüchtlingshilfe. Konfliktdynamik, 1, 40-49.

Stryker, S. \& Burke, P. J. (2000). The past, present, and future of an identity theory. Social Psychology Quarterly, 63, 284-297.

Tyler, T. R. \& Blader, S. L. (2003). The group engagement model: Procedural justice, social identity, and cooperative behavior. Personality and Social Psychology Review, 7(4), 349-361.

Thiel, K. (in Druck). Organisation, Motivation und Konflikte in der Freiwilligenarbeit. Eine organisationspsychologische Analyse freiwilligen Engagements in Non-Profit-Organisationen. Katholische Universität Eichstätt-Ingolstadt: Dissertation.

Thiel, K., Strubel, I. T. \& Kals, E. (2016). Engagement aus Empörung? Emotionen als motivationale Grundlagen freiwilliger Engagements. In I. T. Strubel \& Kals, E. (Hrsg.), Freiwilligenarbeit und Gerechtigkeit (S. 101-115). Zürich: Zentrum für Organisations- und Arbeitswissenschaften EHTZ.

Van Schie, S., Güntert, S. T., Oostlander, J. \& Wehner, T. (2014). How the organizational context impacts volunteers: A differentiated perspective on self-determined motivation. VOLUNTAS: International Journal of Voluntary and Nonprofit Organizations, 26(4), 1570-1590.

Van Schie, S., Güntert, S. T. \& Wehner, T. (2015). Gestaltung von Aufgaben und organisationalen Rahmenbedingungen in der Freiwilligenarbeit. In T. Wehner \& S. T. Güntert (Hrsg.), Psychologie der Freiwilligenarbeit. Motivation, Gestaltung und Organisation (S. 131-149). Berlin: Springer.

Wehner, T. \& Güntert, S. T. (Hrsg.). (2015). Psychologie der Freiwilligenarbeit. Motivation, Gestaltung und Organisation. Berlin: Springer.

Wehner, T. \& Güntert, S. T., Neufeind, M. \& Mieg, H. A. (2015). Frei-gemeinnützige Tätigkeit: Freiwilligenarbeit als Forschungs- und Gestaltungsfeld der Arbeits- und Organisationspsychologie. In T. Wehner \& S. T. Güntert (Hrsg.), Psychologie der Freiwilligenarbeit. Motivation, Gestaltung und Organisation (S. 3-22). Berlin: Springer. 
Preprints 2021 der Zeitschrift BIBLIOTHEK - Forschung und Praxis, BFP.2020.0103 Kals, Strubel und Güntert

Dies ist ein Preprint $(\boldsymbol{C c})$ EY-NC-ND. Die endgültige Publikationsfassung erscheint beim Verlag De Gruyter unter https://www.degruyter.com/view/j/bfup

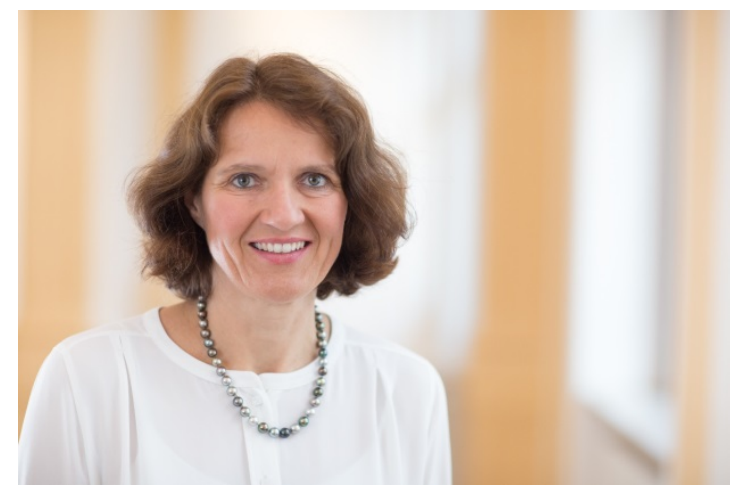

Prof. Dr. Elisabeth Kals

Professorin für Sozial- und Organisationspsychologie

Katholische Universität Eichstätt-Ingolstadt

D-85072 Eichstätt

elisabeth.kals@ku.de

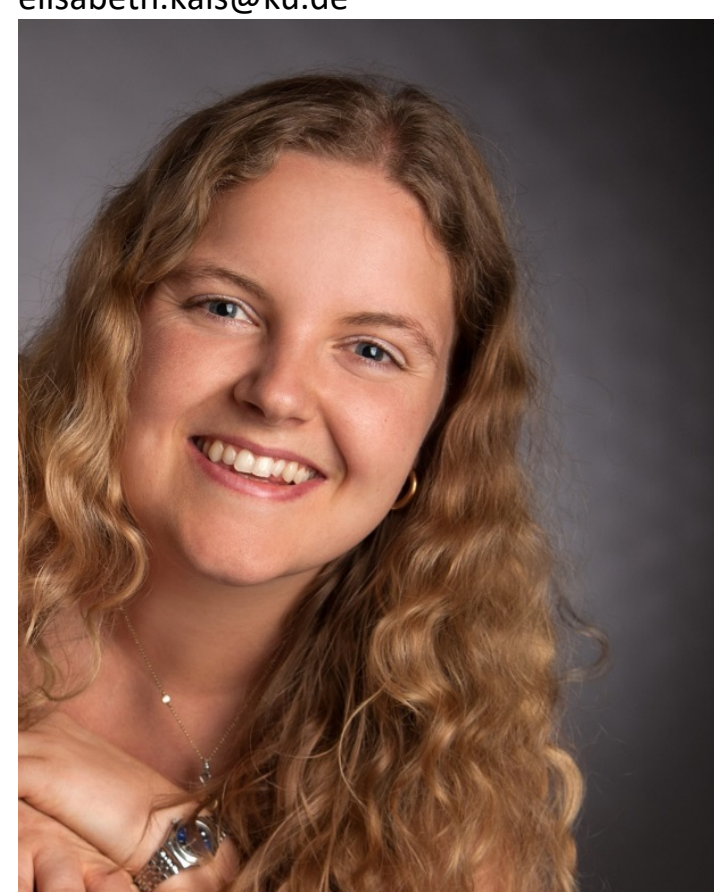

\section{Dr. Isabel Theresia Strubel}

Sozial- und Organisationspsychologie Katholische Universität Eichstätt-Ingolstadt D-85072 Eichstätt isabel.strubel@gmail.com

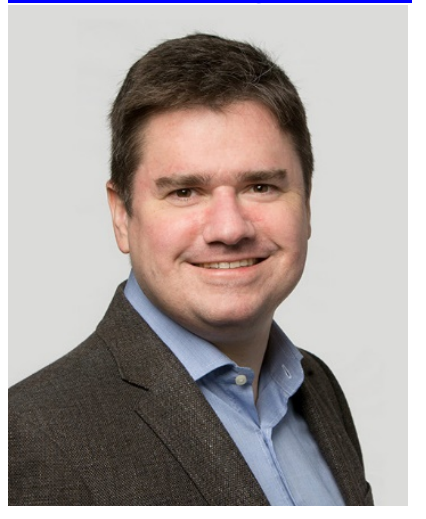


Preprints 2021 der Zeitschrift BIBLIOTHEK - Forschung und Praxis, BFP.2020.0103 Kals, Strubel und Güntert

Dies ist ein Preprint $(\mathrm{cc})$ EY-NC-ND. Die endgültige Publikationsfassung erscheint beim Verlag De Gruyter unter https://www.degruyter.com/view/j/bfup

\section{Dr. Stefan T. Güntert}

Institut für Nonprofit- und Public Management

Hochschule für Wirtschaft

Fachhochschule Nordwestschweiz

Peter Merian-Strasse 86

$\mathrm{CH}-4002$ Basel

Schweiz

stefan.guentert@fhnw.ch 